\title{
Choice reactions with a null stimulus event ${ }^{1}$
}

A procedure for producing a null stimulus $\left(S_{0}\right)$ in a CRT task is described. The $S_{0}$ produced is shown to elicit reactions that have characteristics of responses given to stimuli $\left(S_{1}\right)$ that appear in regular CRT tasks. It was also discovered that, in this procedure, the initiation of $S_{0}$ sampling comes earlier than that of $S_{1}$ when $S_{0}$ and $S_{1}$ appear in the same $C R T$ task. The nature of $S_{0}$ as well as the apparent reasons for its early sampling are discussed.

One of the purposes of this paper is to describe a procedure by which a nonappearing or "null" stimulus can be presented in a CRT task. The existence of a null stimulus $\left(S_{0}\right)$ produced by this method is strongly intuitive, but we will endeavor to describe what we feel are some of the other possible stimulus events to which $S$ responds. In addition, an investigation will be made of the sampling characteristics of $S_{0}$.

The method used to produce $S_{0}$ can be described as a countdown procedure. A string of warning signals, given in beats at regular intervals, is followed by the stimulus event (either $S_{0}$ or $S_{1}-$ a non-null stimulus) that appears in rhythm with the countdown. A predictable point (or small interval) in time is thus created at which $S_{1}$ is or is not given. If the $S$ is instructed to react on every trial, he would not wait indefinitely before producing a response when $S_{0}$ is "presented." The fact that, under these conditions, the $S$ would react on every trial leads us to consider to what he would be responding if no stimulus is given. There are two distinct and opposing ways in which the $S$ can respond during an $S_{0}$ trial. The first is that $S_{0}$ is truly created and it is seen as one of two stimulus events to which one of two CRT responses should be made. The second is that the $S$ may note the interval of time measured from the last countdown signal and regard its length as something to be judged then indicated by one of two possible overt responses. This, then, is the first task of the ensuing experiment-to indicate whether the $S$ is making a choice or is judging an interval of time and no more.

In order to resolve this question, two experimental procedures must be compared, both of which would present $S_{0}$ or $S_{1}$ after a countdown sequence. In one, the $S$ would be given standard CRT instructions. In the other, however, the primary task for the $S$ would be to estimate the size of the interval between the successive countdown stimuli, then make one kind of response at the end of that interval, as measured from the last countdown signal. He would be told that on half of the trials, a stimulus would appear after the last countdown signal, and that on these trials a different response should be made. This task will be called an interrupted interval-estimation (IIE) procedure.

It is important to note that the stimulus events, the interval judgment, and the overt responses required of the $S$ are the same in both the CRT task employing $S_{0}$ and the IIE procedure. Only the processing of presented information is different. In the first instance, a choice is made; in the second, it is not.

The insertion of a choice into the task has the consequence of imposing a variable upper bound on the response-time band associated with an otherwise pure interval-estimation event. This variable upper bound is probabilistic and follows logically from theoretical accounts of RT that incorporate an accumulator, or "counting mechanism," such as that proposed by McGill (1963). The accumulation of decision-making information will eventually reach a criterion, but when this limit will be reached is determined by a probability and not by a fixed time. The skewed distribution that is likely to result from a variable upper bound has practically become a defining characteristic of reaction time (McGill \& Gibbon, 1965).

With an interval estimation requirement, the upper bound to the response-time band would be fairly rigid. That is, there would be a point beyond which the S's estimation of the interval would not exceed, and this point would perhaps be fairly stable. Because this upper bound might be reasonably fixed, normally distributed time-interval-estimation responses would seem to be likely possibilities.

The possible difference in the measure of skewness of these distributions motivates the following hypothesis, which can be stated in three parts. (1) The IIE procedure should produce interval-estimation-response distributions that are no different in degree of skewness than the response times found in making simple, uninterrupted interval estimations. (2) The reaction time distributions for $S_{0}$ in an $S_{0}$-CRT task should be as skewed as the reaction time distributions for $S_{1}$ in a normal CRT procedure. (3) The reaction time in choice tasks and the response times found in time estimations should differ significantly in their measures of skewness. If these three conditions are met, then it may be logically inferred that the response to $S_{0}$ more resembles a response to a stimulus than an estimation of an interval.

Hypotheses concerning the nature of $S_{0}$ sampling are difficult to come by without first looking at the data. For this reason, this question will be approached empirically.

\section{Subjects and Design \\ METHOD \\ An intact groups design was used with} four independent groups and eight Ss (university students) in each group. The two treatments were an interval-estimation procedure and a choice procedure. The interval-estimation procedure, given to two groups, included the IIE task described above and an uninterrupted interval-estimation (UIE) task. The choice procedure used two groups of Ss, both given a two-choice CRT task. In one group, two non-null stimuli $\left(S_{r}\right.$ and $S_{g}$ ) were presented, and in the other group one of the stimulus events was null.

\section{Apparatus}

The essential components of the apparatus were a display of stimulus lights, a three-position lever switch, a Hunter Klockounter, and programming equipment to control the presentation of the countdown signals and stimuli. The lights were two vertical columns of programming equipment indicator lamps (LVE Model 1356-12). The six lamps in the left column comprised the six countdown signals, and two stimulus lights were located in the middle of the right-hand column. The countdown lights were white, and they flashed sequentially at $1 / 2-\sec$ intervals during the foreperiod. The two stimulus lights were red $\left(S_{r}\right)$ and green $\left(\mathrm{S}_{\mathrm{g}}\right)$, and, if given, a stimulus light appeared $1 / 2$ sec after the last countdown signal. The two responses required of the $S$ were movements of the lever switch to one side or the other from the middle position. 


\section{General Procedure}

The response requirements were counterbalanced across Ss so that the responses demanded of each $S$ for the stimulus events were the same across all trials. The eight combinations of the two countdown directions ( $t-b$ or $b-t)$, the two positions of the two stimuli $(t-b$ or $b-t)$, and the two stimuli presented $\left(S_{0}\right.$ or $S_{1} ; S_{r}$ or $S_{\mathrm{g}}$ ) were evenly divided among the trials given to each $S$ by randomly assigning them to the eight trials in each eight-trial block. Twelve blocks of trials were presented, giving a total of 96 trials. The S enjoyed a brief rest after the first 48 trials. Speed of response was emphasized to each $S$ regardless to which group he belonged. Where applicable, the $S$ was informed of the errors he made.

\section{UIE Group}

Each $S$ in this group was given 96 trials of an interval-estimation task. No stimulus appeared after the last countdown light. They were told that the countdown lights would flash at $1 / 2$-sec intervals, and that they were to push the switch in the appropriate direction $1 / 2 \mathrm{sec}$ after the last countdown light was given.

\section{IIE Group}

For these Ss, no stimulus light appeared on half of the trials in each block of eight. In the remaining trials, a red or green stimulus light appeared. The Ss were told that their primary task was to estimate an interval of time, i.e., to push the switch in the appropriate direction in rhythm to the countdown lights so that the response was made at $1 / 2 \mathrm{sec}$ after the last countdown light. After $\mathrm{E}$ was confident that the $S$ understood this instruction, he told the $S$ that an additional task would be required. On some trials, the $S$ was told, a stimulus light would come on after the last countdown signal, at which time he was to push the switch in a direction opposite to what indicated the end of the $1 / 2-\sec$ interval.

\section{$S_{0}$-CRT Group}

The Ss in this group and those in the IIE group were treated essentially the same; they differed only in instructions. Here the Ss were told that, following the countdown warning signals, a red or green light would come on or no light would appear. If a light appeared, they were to push the

Fig. 1. Means of the median RTs to $S_{0}$ and $S_{1}$ given in each block of eight trials. The RTs were measured from the presentation of $s_{1}$. The broken-line function is the average of the two CRT functions shown in Fig. 2. switch in one direction, and if no light appeared, they were to push the switch the other way.

\section{CRT Group}

The Ss in this group received 96 trials, on half of which a green light was given and on the remaining half of which a red light appeared at the appropriate time following the countdown sequence. Again, the division was random within each block of eight trials. The Ss were instructed to make one response when the red light appeared and the other when a green light was given.

\section{RESULTS}

It should be noted that the Ss made appropriate use of the metered countdown lights. This was evidenced by body movements made by most 'Ss in rhythm to the countdown signals (a tapping foot or bobbing head). The presence of these movements also speaks for S's motivation to perform rapidly during the task. He was not told to make such motions or to overtly count the metered signals; these rhythmic responses seemed to arise spontaneously.

In all groups, the Ss' responses seemed to stabilize or reach an asymptotic level by the fifth or sixth block of eight trials. This is clearly shown in Figs. 1 and 3. Consequently, the data from the first 32 trials were not used in these analyses.

The UIE data indicated that the Ss' estimations fell short of the $1 / 2-\sec$ interval measured from the last countdown signal, but that they were fairly reliable in their judgments. To remain in keeping with later analyses, the median RTs for each of the eight blocks of eight trials from Trial 33 were taken as the basic data. The 64 measures produced a mean time estimation of $482 \mathrm{msec}$. The average estimations ranged from 467 to $495 \mathrm{msec}$ across trial
Table 1

Mean Measure of Skewness Across Subjects for the Response Time Distributions in Each of the Four Groups

\begin{tabular}{|c|c|c|c|}
\hline \multicolumn{2}{|c|}{$\begin{array}{c}\text { Time Estimation } \\
\text { Procedure }\end{array}$} & \multicolumn{2}{|c|}{$\begin{array}{c}\text { Choice } \\
\text { Procedure }\end{array}$} \\
\hline $\begin{array}{l}\text { UIE } \\
\text { Group } \\
\end{array}$ & $\begin{array}{c}\text { IIE } \\
\text { Group }\end{array}$ & $\begin{array}{c}\text { So-CRT } \\
\text { Group }\end{array}$ & $\begin{array}{c}\text { CRT } \\
\text { Group }\end{array}$ \\
\hline-.006 & .060 & .530 & .670 \\
\hline
\end{tabular}

blocks, giving a trial-block-to-trial-block accuracy of \pm 15 msec from the mean.

For each $S$ in each of the four major conditions, the distributions of the following RT responses were made: UIE group-all responses from Trials 33-96, inclusive; IIE group Ss-the interval-estimation responses only; $S_{0}-C R T$ group $S s-$ the responses from the $S_{0}$ trials only; CRT group Ss-all responses to $S_{r}$ and $S_{g}$ taken independently. A measure of skewness based on the second and third moments $\left(g_{1}\right)$ was computed for each distribution. Since the responses of each $S$ in the CRT group were made to two different non-null stimuli, the skewness of the distributions found for both sets of responses were ascertained, and a correlated $t$ test was made between the means of the distributions of these two skewness measures across Ss. This test indicated that the differences between these two means did not exceed that expected by chance $[t(7)=1.258$, $p>.20]$. Consequently, the mean of the measures of skewness for the $S_{r}$ and $S_{g}$ pair of distributions were figured for each $S$ and taken as the basic data for this condition.

Table 1 gives the means of these distributions of skewness for the four groups of Ss. In the analysis of variance for this table, the groups-within-treatments $F$ value was less than 1 , indicating that the difference between Groups UIE and IIE and the separation between the $S_{0}$-CRT

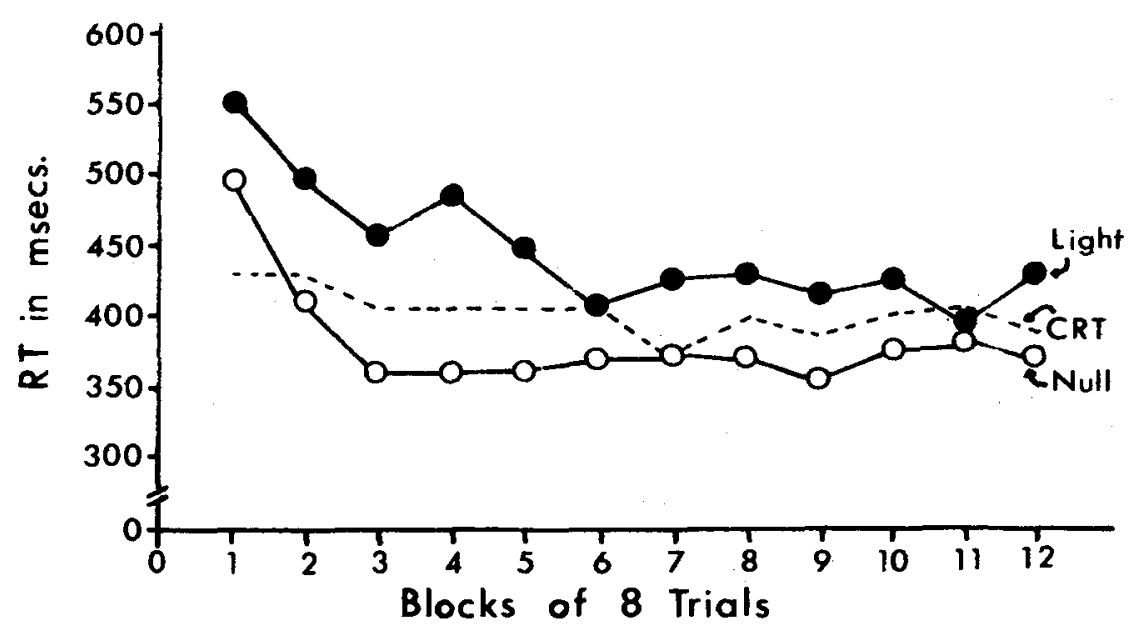




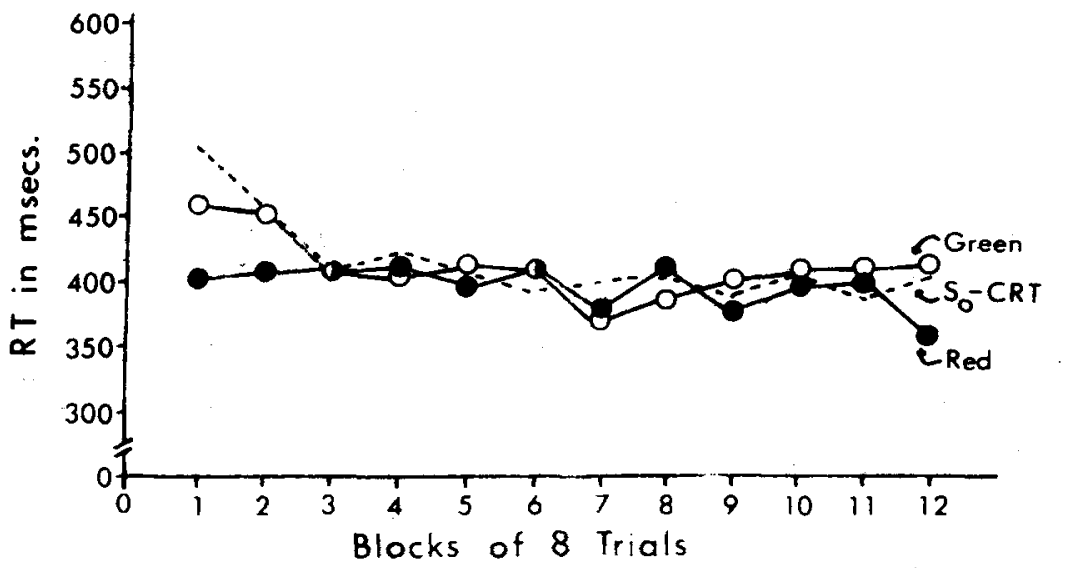

group and the CRT Ss could be ignored. On the other hand, the differences in skewness between the time-estimation tasks and the choice tasks were highly significant $[F(1,28)=7.900, p<.01]$.

Evidence relating to the sampling characteristics of $S_{0}$ might come from an investigation of the RT central tendencies in these conditions. Therefore, comparisons between the RTs to both stimulus events in each of the IEE, $\mathrm{S}_{\mathrm{0}}-\mathrm{CRT}$, and CRT groups were made. Because of the counterbalancing, both stimulus events $\left(S_{0}\right.$ and $S_{1}$ or $S_{x}$ and $S_{g}$ ) were presented on a randomly chosen set of four trials within each eight-trial block. The data selected for analysis were the median RTs for the two stimulus sets within each of these blocks. The means across Ss of these median scores are plotted in Figs. 1, 2, and 3.

An analysis of variance for a completely within-Ss design was used to assess the effects of the $S_{0}$-CRT condition only (Fig. 1). Inspection of Figs. 2 and 3 suggested that any statistical analyses of these data would be gratuitous. The analysis of variance indicated that the separation between the $S_{0} R T$ and $S_{1} R T$ was not due to chance $[F(1,7)=18.778$, $\mathrm{p}<.011$. Only the data from Trial Block 5 on were used to see if these two functions might be parallel. The Trial by Stimulus interaction was not statistically significant, nor were the differences from trial to trial. This suggests the possibility that not only were the functions parallel but that they were also asymptotic.

\section{DISCUSSION}

The results suggest that the reaction to $S_{0}$ was something other than merely an estimation of an interval of time. The insertion of choice into the task caused the $S$ to regard $S_{0}$ as a genuine stimulus to which he must respond. The answer to what constitutes this stimulus is elusive. One is tempted to suggest that the estimated time interval itself is the stimulus. If this is true, then at what point does the $S$ begin to sample? It would seem to make little sense to say that he starts to sample in the middle of the interval he is estimating. If he begins to sample at the onset of this interval, then he would be prepared to produce an $S_{0}$ response at the end of the estimated interval, and his $S_{0}$ RT would be less than Fig. 1 indicates. We

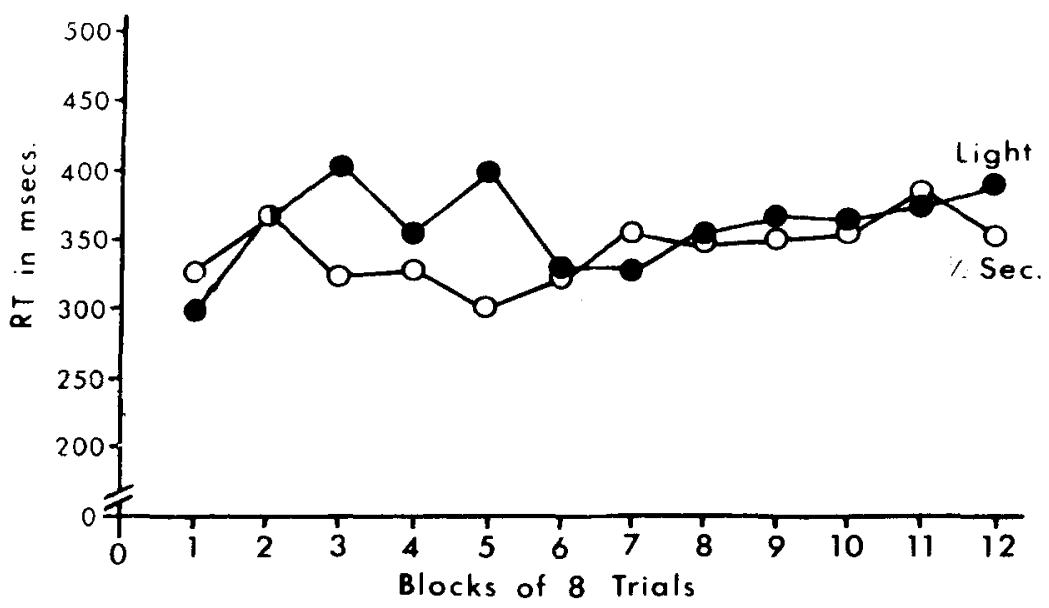

Fig. 2. Mean of the median RTs to $S_{r}$ and $S_{g}$ given in each block of eight trials. The RTs were measured from the presentation of $s_{1}$. The broken-line function is the average of the two $S_{0}-C R T$ functions shown in Fig. 1.

might suggest, then, that the $S$ delays $S_{0}$ sampling until the end of the estimation of the interval. This eliminates the time interval per se as representing the $S_{0}$ event, but it leaves the termination of the interval as a possibility. We will return to this point shortly.

The differences that exist between the $S_{0}$ RTs and the $S_{1}$ RTs shown in Fig. 1 are substantial. The third function in this figure suggests that perhaps the $S_{0} R T$ is suppressed below that of the standard CRT response and that the presence of $S_{0}$ elevates the $S_{1} R T$ above the normal CRT response by an equal amount. The most parsimonious explanation for this result is that the initiation of $S_{0}$ sampling precedes that of $S_{1}$. This seems to run contrary to the discussion in the preceding paragraph since $S_{1}$ would mark the termination of the $1 / 2$-sec interval, and the $S$ would begin to sample $S_{0}$ sometime before the interval terminated. On the $S_{1}$ trials, the $S$ would be given the equivalent of two successive stimuli with instructions to respond to the first of the two $\left(S_{0}\right)$ if, and only if, $S_{1}$ did not occur. These results are in complete agreement with those of Bernstein and Segal (1968) and Yellot and Hildreth (1969), who independently investigated the problems of RT to successive stimuli. The effect of successive presentation of stimuli on RT responses reported here is also in keeping with the predictions of RT theories that postulate response criteria based on accumulated stimulus information (Stone, 1959; McGill, 1963). Suppose $S_{0}$ information was sampled and accumulated to a point somewhat below the criterion for response, at which time $S_{1}$ was presented. The $S$ would have to sample enough $S_{1}$ to override the accumulated $S_{0}$ information as well as reach the $S_{1}$ response criterion cut-off. Thus, long RTs would be expected for the second of the two successive stimuli. Shorter RTs would be expected for the first of the two stimuli since the choice is removed from the procedure, leaving only a simple RT task for the $S$.

Evidence for the validity of this position

Fig. 3. Mean of the median response times given by the $1 / 2-\mathrm{sec}$ interval estimation and the $R T$ to the interrupting stimulus. The units on the ordinate represent times from the presentation of the interrupting stimulus. 
comes from the analysis of errors. If the $S$ samples $S_{0}$ early enough, then he may be ready to respond to $S_{0}$ when $S_{1}$ appears, and more errors on $S_{1}$ would result. As it happened, $2 \%$ and $6 \%$ errors were produced during the $S_{0}$ and $S_{1}$ trials, respectively. Unfortunately, the CRT group also produced a $6 \%$ error rate, and the fact that the $S_{1}$ trials matched this rate, while the $S_{0}$ trials produced fewer errors, causes the depression of the $S_{0}$ RTs to be suspect.

Suppose the $\mathbf{S}$ was predisposed toward anticipating his $S_{0}$ responses. That is, on a certain proportion of the trials, he responds to $S_{0}$ without having enough $S_{0}$ information to satisfy the $S_{0}$ response criterion. These anticipatory responses would be seen (as errors) in the $S_{1}$ trials only. Since their appearance in the $S_{0}$ trials would not be apparent, the anticipatory responses would be included in the $S_{0}$ data and, by virtue of their anticipatory nature, would depress the average $S_{0} R T$. The presence of a low error rate in general argues that the average would not be diminished significantly by so few anticipatory responses. In any event, an attempt was made to compensate for possible decreased RTs produced by anticipation in the $S_{0}$ trials. The RTs in both the $S_{0}$ and $S_{1}$ trials were ranked for each $S$. On the supposition that the anticipation responses in the $S_{0}$ trials would be made at the same speed as the anticipation responses caught in the $S_{1}$ trials, each $S_{0} R T$ of a rank corresponding to an $S_{1}$ error was eliminated and the data recomputed. The results were no different from the ones shown here.

Since $S_{0}$ sampling precedes the end of the $1 / 2$-sec interval from the last countdown signal, the question now becomes how much before the end of the interval does the sampling begin. At least two possible answers are suggested by these data. The first is that the $\mathbf{S}$ keeps pushing his point of initial sampling further back into the $1 / 2-\sec$ interval until the error rate becomes intolerable (6\%?). The second is that the $S$ begins to sample at the end of his estimated $1 / 2-\sec$ interval, which, in a simple interval-estimation task, is $482 \mathrm{msec}$. The second of these two explanations allows us to assume that the $S$ samples $S_{0}$ prior to $S_{1}$, yet his $S_{0}$ sampling can begin with the termination of the postcountdown interval (as he sees it). However, of the two possibilities, the first suggestion, that the $S$ will tolerate a small error rate, seems to receive more support from the data. We do not know that the $1 / 2$-sec interval estimation under circumstances more resembling a CRT task would produce a more accurate estimation of the interval. In fact, the IIE-condition data suggest that indeed it does. One might expect that, if anything, the interval estimation in this IIE task would match the 482-msec estimation of the UIE group. If it did, however, then the interval estimation should be clearly less than the response times to the interrupting stimulus, which was presented at the 500-msec mark. As Fig. 3 shows, they were virtually the same after they became stable at the sixth trial block. These functions also rise curiously, a phenomenon we will not pursue here but one that is worthy of further study.

\section{REPERENCES}

BERNSTEIN, I. H., SEGAL, R. Set and temporal integration. Perception \& Psychophysics, 1968, 4, 233-236.

MCGILL, W. J. Stochastic latency mechanism. In R. D. Luce, R. R. Bush, and E. Galanter (Eds.), Handbook of mathematical. psychology. Vol. 2. New York: Wiley, 1963. Pp. 309-360.

MCGILL, W. J., \& GIBBON, I. The general gamma distribution and reaction times. Joumal of Mathematical Psychology, 1965, 2 , 1-18.

STONE, M. Models for choice reaction time. Psychometrika, 1960, 25, 251-260.

YELLOTT, J., \& HILDRETH, J. How long does it take to see red? Paper presented at the Midwestern Mathematical Psychologists' meeting held at Bloomington, Indiana.

\section{NOTES}

1. This research was supported by the Oklahoma State University Research Foundation and was undertaken while the second author was an NDEA Fellow. Different parts of the data were reported at the 1968 Psychonomic Society meetings in St. Louls and at the 1969 Mid-western Psychologists' meeting held at Bloomington, Indiana.

2. Address: Oklahoma State University, Stiilwater, Oklahoma 74074.

(Accepted for publication July 17, 1969.) 